\title{
Conductance in single electron transistors with quantum confinement
}

\author{
F. O. Heinz ${ }^{*}$, A. Schenk, and W. Fichtner \\ Institut für Integrierte Systeme, ETH Zentrum, 8092 Zürich, Switzerland
}

Received 30 September 2002, accepted 2 December 2002

Published online 23 June 2003

PACS 05.10.Ln, 73.23.Hk

Single electron transistors are studied by self-consistent 3D quantum mechanical simulation. Computation of the linear-response conductance requires the calculation of thermal ensemble averages. The evaluation of these quantities can be substantially accelerated by means of Monte-Carlo sampling.

1 Introduction A single electron transistor (SET) is a three terminal device without a classically conducting path from source to drain: between source and drain resides a capacitatively gated island with tunnel junctions on either side (cf. Fig. 1). As long as the dimensions of the island regions are large compared with the electron thermal wavelength, an SET may be readily described in terms of the 'orthodox theory' [1]. For islands with dimensions comparable to the electron thermal wavelength, however, quantum confinement effects and energy-dependent tunnelling rates render the orthodox theory inapplicable. It is these quantum dot SETs that the present paper addresses. Computation of the conductance of such devices calls for a microscopic approach, that accounts correctly for both the quantum mechanics and the statistical mechanics of the quantum dot and its surroundings.

2. Simulation Method Our simulation approach is based on the self-consistent evaluation of the electronic structure inside a 3D geometry model of the device under study [2]. The simulation volume

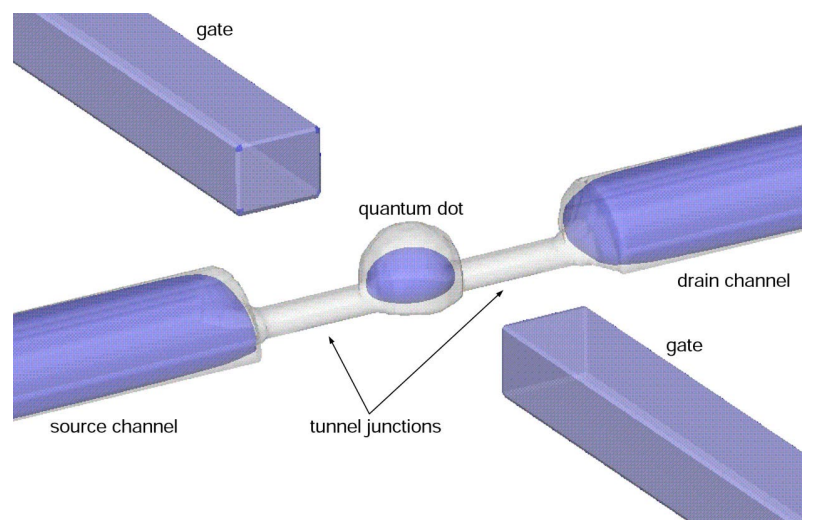

Fig. 1 (online colour at: www.interscience. wiley.com) Simulation geometry of a nanoscale SOI SET with charge density iso-surface.

\footnotetext{
* Corresponding author: e-mail: frederik.heinz@iis.ee.ethz.ch, Phone: +41 1632 59 57, Fax: +41 16321194
} 
is sub-divided into quantum well, quantum wire and quantum dot regions depending on the degree of confinement along the different spatial directions. For each region we solve the finite temperature generalisation [3] of the effective mass Kohn-Sham equations in local density approximation (LDA). Bardeen's transfer Hamiltonian method [4] is invoked for the computation of tunnelling rates $\Gamma_{i}^{\mathrm{src}}$ $\left(\Gamma_{i}^{\mathrm{drn}}\right)$ between source (drain) region and the $i$-th single particle level of the quantum dot. In the presence of low potential barriers a 3D generalisation [5] of the transfer Hamiltonian is used for the quantum dot region; this allows us to apply Dirichlet boundary conditions at the surface of an (enlarged) Schrödinger domain without running the risk of introducing spurious states in artificial potential wells near the domain walls.

For each value of the gate voltage, these computations are carried out for various electron numbers $N$ on the quantum dot, giving rise to $N$-dependent Kohn-Sham orbital energies $\epsilon_{i, N}$ and tunnelling rates $\Gamma_{i}^{\mathrm{src} / \mathrm{drn}}(N)$, as well as $N$-dependent Hartree $\left(E_{e-e}(N)\right)$, exchange-correlation $\left(E_{\mathrm{xc}}(N)\right)$ and exchange-correlation potential $\left(E_{\mathrm{xc}-\text { pot }}(N)\right)$ energies.

3 Conductance extraction If the lifetime of the (quasi-)localised quantum-dot states exceeds the inelastic scattering time inside the quantum dot, the dominant mechanism for charge transport from source to drain is sequential tunnelling through the quantum dot. In this transport regime, the linear response conductance of the device is given by Beenakker's conductance formula [6]:

$$
\begin{aligned}
G & =\frac{e^{2}}{k_{\mathrm{B}} T} \sum_{\left\{n_{i}\right\}} P\left(\left\{n_{i}\right\}\right) \sum_{i} \frac{\Gamma_{i}^{\mathrm{src}} \Gamma_{i}^{\mathrm{drn}}}{\Gamma_{i}^{\mathrm{src}}+\Gamma_{i}^{\mathrm{drn}}} \delta_{n_{i}, 0} f\left(\frac{\epsilon_{i, N}^{\prime}-\epsilon_{\mathrm{F}}}{k_{\mathrm{B}} T}\right) \\
& =\frac{e^{2}}{k_{\mathrm{B}} T} \sum_{N} P(N) \sum_{i}\left(1-P\left(n_{i}=1 \mid N\right)\right) \frac{\Gamma_{i}^{\mathrm{src}} \Gamma_{i}^{\mathrm{drn}}}{\Gamma_{i}^{\mathrm{src}}+\Gamma_{i}^{\mathrm{drn}}} \delta_{n_{i}, 0} f\left(\frac{\epsilon_{i, N}^{\prime}-\epsilon_{\mathrm{F}}}{k_{\mathrm{B}} T}\right) .
\end{aligned}
$$

Here $\epsilon_{i, N}^{\prime}$ is the energy needed to populate the $i$-th single electron orbital in a given configuration. $P(N)$ is the probability for having a total electron number of $N$ on the quantum dot; $P\left(n_{i}=1 \mid N\right)$ is the $N$-particle Gibbs distribution, i.e. the probability for occupation of single electron orbital $i$ provided that the total electron number on the quantum dot is $N$.

The $P\left(n_{i}=1 \mid N\right)$ are identical to the phase space averages $\left\langle n_{i}\right\rangle_{N}$ over the $N$-particle sub-space (subsequently denoted as $\left\{n_{i}\right\}_{N}$ ) of the internal phase-space of the quantum dot. Likewise, the $P(N) \propto \exp (-\beta(F(N)-N \mu))$ may be expressed in terms of phase-space averages $\langle\cdot\rangle$, by writing the free energy as

$$
\begin{aligned}
& F(N)=\langle E\rangle_{N}-T \cdot S(N), \\
& S(N)=-k_{\mathrm{B}} \sum_{\left\{n_{i}\right\}_{N}} P\left(\left\{n_{i}\right\} \mid N\right) \log P\left(\left\{n_{i}\right\} \mid N\right)=-k_{\mathrm{B}}\left\langle\log \left(P\left(\left\{n_{i}\right\} \mid N\right)\right)\right\rangle_{N} .
\end{aligned}
$$

In order to make the task of phase-space averaging tractable, constant interaction is assumed inside each $N$-particle segment of phase-space, i.e. $E_{e-e}, E_{\mathrm{xc}}$ and $E_{\mathrm{xc}-\text { pot }}$ depend only on $N=\sum_{i} n_{i}$, not on the specific single-particle configuration $\left\{n_{i}\right\}$. Thus, the difference in energy between two single particle configurations $\left\{n_{i}\right\}$ at equal $N$ reduces to the difference in Kohn-Sham orbital energy $E_{\text {orbital }}\left(\left\{n_{i}\right\}\right)=\sum_{i} n_{i} \epsilon_{i, N}$.

Still, the computational effort for direct evaluation of $\langle\cdot\rangle_{N}$ will be prohibitive for large electron numbers, especially at elevated temperature. Then, the number of single-particle orbitals \#orb that need to be taken into account will be considerably larger than the number of electrons, thus making the number of possible configurations, card $\left(\left\{n_{i}\right\}_{N}\right)=\left(\begin{array}{c}\# \text { orb } \\ N\end{array}\right)$, very large. This has given rise to approximations, e.g. restriction to a $T=0$ ground-state ("Slater rule") or replacement of the free energy $F$ with the internal energy $E$ and use of a shifted Fermi function (with $\tilde{\epsilon}_{\mathrm{F}}$ chosen such that the total electron number $N$ is reproduced) instead of the Gibbs distribution. 
We have developed a Monte-Carlo (MC) sampling scheme that allows for full evaluation of the statistical mechanics of the quantum dot at moderate computational effort even in the case of very large phase spaces. This allows us to study the validity of customary approximations.

4 Monte-Carlo sampling scheme The thermal average of a quantity $A$ may be written as

$$
\langle A\rangle_{N}=\sum_{\left\{n_{i}\right\}_{N}} P\left(\left\{n_{i}\right\} \mid N\right) A\left(\left\{n_{i}\right\}\right)=\frac{1}{\operatorname{card}\left(M_{N}\right)} \sum_{\left\{n_{i}\right\} \in M_{N}} A\left(\left\{n_{i}\right\}\right),
$$

provided that the set $M_{N}$ is chosen in such a way, that the number of occurrences of a micro-state $\left\{n_{i}\right\}$ in $M_{N}$ is proportional to $P\left(\left\{n_{i}\right\} \mid N\right)$. It has been shown by Metropolis [7] that this condition is automatically met, if $M_{N}$ is constructed as a sequence of states $s_{1}, s_{2}, \in\left\{n_{i}\right\}_{N}$ according to the following rules:

Transition: $\boldsymbol{s}_{n} \rightarrow \boldsymbol{s}_{n+1}$ :

1. Choose a random state $s \Rightarrow$ energy: $\epsilon(s)$.

2. Accept $s$ as $\boldsymbol{s}_{n+1}$ with probability

$$
P= \begin{cases}1 & \text { if } \epsilon(s)<\epsilon\left(s_{n}\right), \\ \exp \left(-\frac{\epsilon(s)-\epsilon\left(s_{n}\right)}{k_{\mathrm{B}} T}\right) & \text { otherwise }\end{cases}
$$

3. If $s$ is not accepted, go back to step 1.

The finite sub-sequences $M_{N}^{n}:=\left\{\boldsymbol{s}_{1}, \ldots, \boldsymbol{s}_{n}\right\}$ of $M_{N}$ may be used to obtain estimates $\langle A\rangle_{N}^{n}$ for $\langle A\rangle_{N}$. When estimating the error $\left|\langle A\rangle_{N}^{n}-\langle A\rangle_{N}\right|$, it is important to note that the samples $s_{i}$ are correlated. Therefore, the error is augmented by a factor of $\sqrt{2 \tau+1}$ relative to the uncorrelated case, $\tau$ being the correlation "time" of the sequence $A\left(s_{i}\right)$. In our implementation, only transitions that transfer a single electron to a different orbital are considered. This results in typical correlation times of about 4 sequence steps; the worst correlation time observed was about 50. A less restrictive transition matrix might help reduce correlation times and thus speed up convergence. But, in contrast to the deterministic evaluation of phase space averages, the Monte-Carlo scheme never dominated the total simulation time; thus, little need was felt for optimisation at this stage.

5 Results The results in this section were obtained from simulations modelled on a GaAs split-gate SET manufactured at the University of Würzburg. The effect of Fermi level pinning due to surface states was incorporated into the model as a Dirichlet condition on the conduction band edge at the
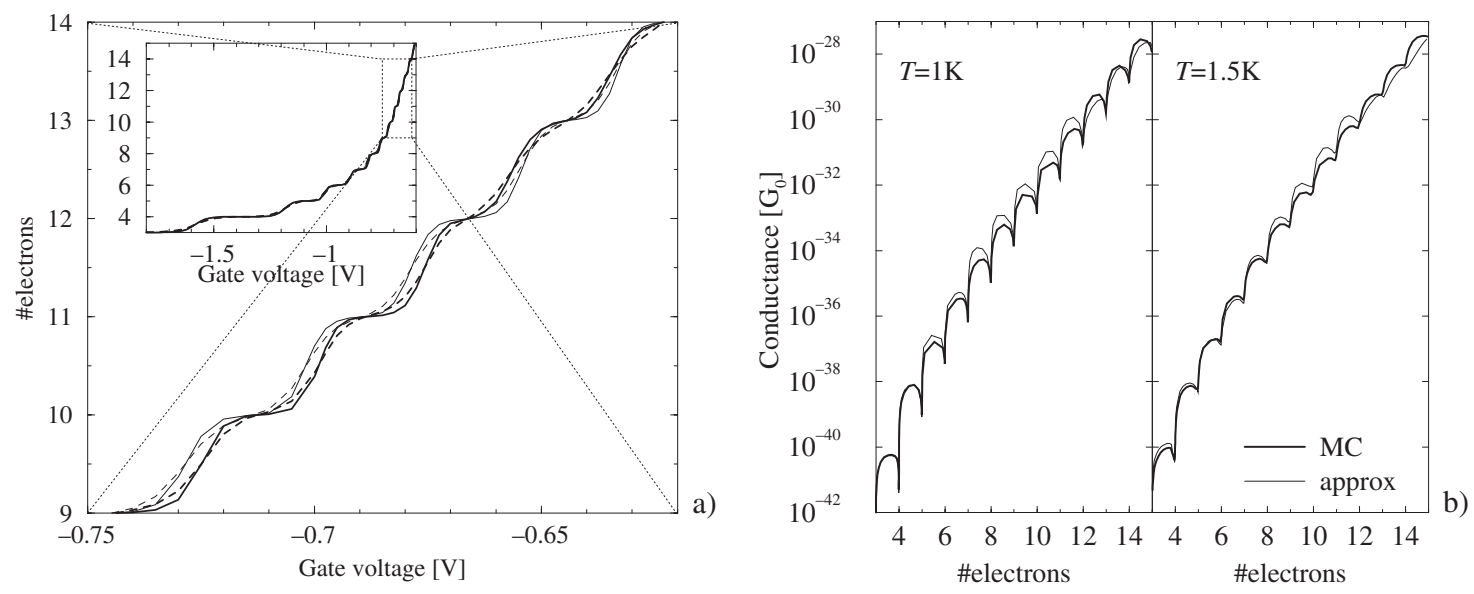

Fig. 2 a) Charging curve of a GaAs SET. -: Monte-Carlo; - : $S=0$. Solid line: $T=1 \mathrm{~K}$; dashed: $T=1.5 \mathrm{~K}$. b) Conductance as function of the electron number. 
exposed GaAs surface. The value of the pinning energy was determined by matching the turn-on voltage of the quantum point contact (QPC) that forms, when the source-dot tunnelling barrier is lowered, to experimental data.

Figure 2 shows the simulated single electron charging curve and the conductance of this device; conductance was plotted vs. electron number for better visibility. The results of the Monte-Carlo process (displayed with heavy lines) were found to be practically identical (i.e. deviation in the electron number $\leq 0.004)$ to the results of the full phase space sum - the $\left\{n_{i}\right\}_{N}$ in this simulation contained up to 155 million states, thus evaluation of the sum was still possible, albeit time consuming. Light lines are used for displaying the results of the approximate evaluation of Eq. (1), in which entropy was neglected and the Gibbs distribution replaced with a shifted Fermi function. The influence of the approximations on the charging curve is noticeable, but quite small; this is due to the fact, that the entropy term contributes only weakly to the free energy. Conductance is more strongly affected by the approximations: deviations as large as a factor of three (both above and below the actual value) can be observed; the distortion of the peak shapes can be seen even from a logarithmic plot.

The absolute values of the simulated conductance are extremely small. This might be a calibration issue: recent simulations have shown the quantum dot electron number (and hence the SET conductance) to be much more susceptible to changes in the pinning energy than the QPC conductance used in its determination. Thus, with the strong increase in conductance with electron number, a slight modification of the surface pinning energy can give rise to a massive increase in linear response conductance. Alternatively, transport through the quantum dot might be coherent rather then dephasing - preliminary calculations for the coherent mechanism suggest good agreement with experimental data; but further work will be needed to clarify this point.

6 Conclusion When explicit evaluation of the statistical terms in the conductance formula becomes intractable in large phase-spaces, Monte-Carlo sampling yields accurate results at much reduced computational effort. This eliminates the need for ad hoc approximations of questionable validity.

Acknowledgements This work has been carried out in the context of the EU research project IST-1999-10828 (NANOTCAD) with financial support by the Swiss Federal Office for Education and Science (BBW).

\section{References}

[1] K. Likharev, Proc. IEEE 87, 606 (1999).

[2] A. Scholze, A. Schenk, and W. Fichtner, IEEE Trans. Elec. Dev. 47, 1811 (2000).

[3] N. D. Mermin, Phys. Rev. 137, 1441 (1965).

[4] J. Bardeen, Phys. Rev. Lett. 6, 57 (1961).

[5] F. O. Heinz, A. Schenk, A. Scholze, and W. Fichtner, J. Comput. Electron. 1, 161 (2002).

[6] C. W. J. Beenakker, Phys. Rev. B 44, 1646 (1991).

[7] N. Metropolis and S. Ulam, J. Am. Stat. Assoc. 44, 335 (1949). 